\title{
COMMENTARY
}

\section{Functional relationship between Campylobacter concisus and the stomach ecosystem in health and disease}

\author{
Nadeem O Kaakoush, Si Ming Man and Hazel M Mitchell
}

The ISME Journal (2013) 7, 2245-2247; doi:10.1038/ ismej.2013.115; published online 4 July 2013

Our group has read with great interest the article by von Rosenvinge et al. (2013) on the characterization of the microbiota present in the stomach fluid using both 16S ribosomal DNA and ribosomal RNA sequencing. Gastric acidity and proteolytic enzymes act as a barrier to ingested microbes, thereby influencing the microbial ecology of the entire gastrointestinal tract (GIT). This is supported by the findings of von Rosenvinge et al. (2013) and others, who have shown that human gastric fluid harbors a microbiota with similar overall composition at the phylum level to that in other GIT locations. Thus, specific changes in the microbiota of the stomach could potentially be involved in gastric or intestinal manifestations such as Crohn's disease (CD) whose development is influenced by microbial dysbiosis (Man et al., 2011).

In their study, von Rosenvinge et al. (2013) reported the transcriptional activity of members of Actinobacteria to be as low as $34 \%$, while that of Tannerella to be as high as $680 \%$ in patients diagnosed with a range of clinical manifestations, including erythematous gastropathy, gastric ulcers and Barrett's esophagus. This was determined by calculating the ratio of relative $16 \mathrm{~S}$ rRNA abundance over relative 16S rDNA abundance for each bacterial taxon identified. Actinobacteria are a large and ecologically diverse group of bacteria, some of which are pathogenic in humans. To understand their involvement in health and disease, functional studies on the roles of specific members of Actinobacteria with decreased transcriptional activity in the stomach (the oral habitants Rothia dentocariosa and Actinomyces odontolyticus) are required. Nevertheless, the data from von Rosenvinge et al. (2013) would suggest that the Actinomycineae (to which Actinomyces spp. belong) were transcriptionally most active in a patient (patient \#44) with no endoscopic findings (RNA reads/DNA reads $=1.04$ vs 0.13 (patient \#33), 0.27 (patient \#52), 0.37 (patient \#50) and 0.077 (patient \#34)), and transcriptionally more active in patients not on proton pump inhibitors (1.04 and 0.37 vs $0.13,0.27$ and 0.077 ).
Tannerella forsythia has been previously detected in neonatal gastric aspirates (Gonzales-Marin et al., 2011), and is considered a dental pathogen. The precise role of this bacterium in the stomach is less clear, and further work on this pathogen is required. Of particular interest was the finding that Campylobacter species, particularly Campylobacter concisus (Figure 1), were highly active (an increase of $444 \%$ ) within the gastric fluid, irrespective of the $\mathrm{pH}(\mathrm{pH}$ 1.0, 6.5 and 8.5; von Rosenvinge et al., 2013). Previous studies have reported the isolation of $C$. concisus from human intestinal biopsies and fecal samples (Kaakoush and Mitchell, 2012), providing convincing evidence that $C$. concisus has the ability to survive stomach acid regardless of $\mathrm{pH}$ levels. The closely related pathogen C. jejuni induces an adaptive tolerance response when exposed to acid stress, and more importantly C. jejuni remains viable and maintains a helical shape following a 20-min exposure to $\mathrm{pH} 4.5$ (Reid et al., 2008). Thus, it is possible that some C. concisus strains utilize similar mechanisms of acid stress resistance, which enables them to thrive and remain metabolically active in the low $\mathrm{pH}$ levels of the gastric environment.

What role $C$. concisus may play in the stomach and why it is so highly active in this acidic environment is unclear; however, previous studies provide some insights into these questions. Using a C. concisus-specific PCR, we have previously shown a significantly higher prevalence of $C$. concisus DNA to be present in children with newly diagnosed CD $(65 \%, 35 / 54)$ than in healthy controls $(33 \%, 11 / 33$, $P=0.008)$ and non-CD controls (37\%, 10/27, $P=0.03$ ) (Man et al., 2010b). Interestingly, CD affects all sections of the GIT from the oral cavity to the anus, and a large percentage $(89.5 \%)$ of the children with CD in our pediatric studies experienced L4 (upper GIT: esophagus and stomach) involvement (Man et al., 2010b). The association of C. concisus with a form of CD that shows specific involvement of L4 raises the possibility that this pathogen may cause clinical pathology within the stomach.

C. concisus has also been detected in esophageal aspirate specimens and in distal esophageal mucosal samples from patients with Barrett's esophagus (Macfarlane et al., 2007). Further, 3 of 25 patients in 


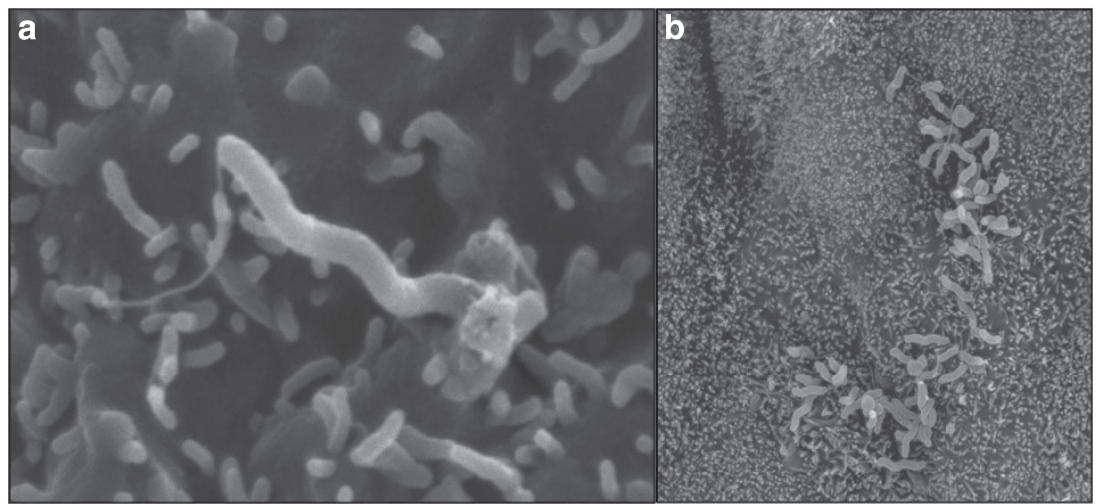

Figure 1 C. concisus is an emerging pathogen of the gastrointestinal tract. C. concisus invades a human intestinal epithelial cell, generating a membrane-ruffling phenomenon on the host cell membrane (a). C. concisus preferentially attaches to the intercellular junction of a human intestinal epithelium (b). Permission obtained from Oxford University Press (C) (Man et al., 2010a).

the study by von Rosenvinge et al. (2013) were diagnosed with Barrett's esophagus. RNA-based amplicons were obtained for one of these patients (patient \#50), and in this patient relative Campylobacter RNA reads were $~ 7.5$ times more than Campylobacter DNA reads. The genetic diversity of $C$. concisus strains has been shown to contribute to the transcellular invasiveness of $C$. concisus, with strains isolated from chronic intestinal diseases being over 500 times more invasive than those isolated from acute intestinal diseases and healthy subjects (Kaakoush and Mitchell, 2012). While in vitro studies have shown that $C$. concisus strains adhere to and invade human intestinal epithelial cells (Kaakoush and Mitchell, 2012), further work is required to investigate whether C. concisus strains can invade epithelial cells of esophageal and gastric origin.

A further interesting finding within the study by von Rosenvinge et al. (2013) was the more pronounced activity of $C$. concisus in an immunocompromised patient as compared with the four samples from immunocompetent patients. C. concisus is highly immunogenic, and we have identified 31 C. concisus proteins to be immunoreactive in children with CD (Kaakoush and Mitchell, 2012). Moreover, patients with CD have elevated levels of $C$. concisusspecific immunoglobulin-G antibodies as compared with controls (Kaakoush and Mitchell, 2012), suggesting that the host immune system may be essential in controlling the levels of $C$. concisus in the GIT.

The functional relationship between $C$. concisus and other members of the stomach mucosa microbiota is unknown. The authors reported that the presence of Helicobacter pylori was not associated with significant differences in C. concisus levels (von Rosenvinge et al., 2013). It is known that the response to $H$. pylori infection differs in individuals, and a subset of patients infected with $H$. pylori experience higher levels of acidity within the stomach while others remain asymptomatic or experience lower acidity (Suzuki and Moayyedi, 2013). Given the ability of $C$. concisus to remain active within a broad $\mathrm{pH}$ range (1.0-8.5) (von Rosenvinge et al., 2013), this may explain the lack of effect observed by $H$. pylori on C. concisus levels.

The findings of von Rosenvinge et al. (2013), and the fact that $C$. concisus colonizes the oral cavity (Kaakoush and Mitchell, 2012), esophagus (Macfarlane et al., 2007), the gastric mucosa (Bik et al., 2006), as well as most areas of the intestines, including the ileum, jejunum, cecum and rectum (Kaakoush and Mitchell, 2012), indicate that this bacterium can colonize almost the entire GIT. Given this, elucidation of the role of commensal and pathogenic $C$. concisus strains within the GIT, the effect of these strains on the GIT microbiota, and the interplay between $C$. concisus strains with different pathogenic potential in the modulation of the host immune response would be of significant interest.

\section{Conflict of Interest}

The authors declare no conflict of interest.

\section{Acknowledgements}

NOK is supported by an Early Career fellowship from the National Health and Medical Research, Australia.

NO Kaakoush and HM Mitchell are at School of Biotechnology and Biomolecular Sciences, The University of New South Wales, Sydney, New South Wales, Australia Si Ming Man is at Department of Veterinary Medicine, University of Cambridge, Cambridge, UK E-mail: H.Mitchell@unsw.edu.au

\section{References}

Bik EM, Eckburg PB, Gill SR, Nelson KE, Purdom EA, Francois F et al. (2006). Molecular analysis of the bacterial microbiota in the human stomach. Proc Natl Acad Sci USA 103: 732-737. 
Gonzales-Marin C, Spratt DA, Millar MR, Simmonds M, Kempley ST, Allaker RP. (2011). Levels of periodontal pathogens in neonatal gastric aspirates and possible maternal sites of origin. Mol Oral Microbiol 26: $277-290$.

Kaakoush NO, Mitchell HM. (2012). Campylobacter concisus-a new player in intestinal disease. Front Cell Infect Microbiol 2: 4.

Macfarlane S, Furrie E, Macfarlane GT, Dillon JF. (2007). Microbial colonization of the upper gastrointestinal tract in patients with Barrett's esophagus. Clin Infect Dis 45: 29-38.

Man SM, Kaakoush NO, Mitchell HM. (2011). The role of bacteria and pattern-recognition receptors in Crohn's disease. Nat Rev Gastroenterol Hepatol 8: 152-168.

Man SM, Kaakoush NO, Leach ST, Nahidi L, Lu HK, Norman J et al. (2010a). Host attachment, invasion, and stimulation of proinflammatory cytokines by Campylobacter concisus and other non-
Campylobacter jejuni Campylobacter species. I Infect Dis 202: 1855-1865.

Man SM, Zhang L, Day AS, Leach ST, Lemberg DA, Mitchell H. (2010b). Campylobacter concisus and other Campylobacter species in children with newly diagnosed Crohn's disease. Inflamm Bowel Dis 16: 1008-1016.

Reid AN, Pandey R, Palyada K, Whitworth L, Doukhanine E, Stintzi A. (2008). Identification of Campylobacter jejuni genes contributing to acid adaptation by transcriptional profiling and genome-wide mutagenesis. Appl Environ Microbiol 74: 1598-1612.

Suzuki H, Moayyedi P. (2013). Helicobacter pylori infection in functional dyspepsia. Nat Rev Gastroenterol Hepatol 10: 168-174.

von Rosenvinge EC, Song Y, White JR, Maddox C, Blanchard T, Fricke WF. (2013). Immune status, antibiotic medication and $\mathrm{pH}$ are associated with changes in the stomach fluid microbiota. ISME $J$ 7: 1354-1366. 